\title{
The Study of a Dual-Disk Type Piezoelectric Actuator
}

\author{
W. M. Chen, C. H. Chan, and T. S. Liu \\ Department of Mechanical Engineering, National Chiao Tung University, Hsinchu 30010, Taiwan \\ Correspondence should be addressed to T. S. Liu; tsliu@mail.nctu.edu.tw
}

Received 30 June 2013; Revised 7 November 2013; Accepted 7 November 2013

Academic Editor: Evangelos J. Sapountzakis

Copyright (C) 2013 W. M. Chen et al. This is an open access article distributed under the Creative Commons Attribution License, which permits unrestricted use, distribution, and reproduction in any medium, provided the original work is properly cited.

\begin{abstract}
This study presents an innovative piezoelectric actuator, capable of long travel distance and fast speed. Main components of the actuator include a rod and two piezoelectric buzzers of disk geometry. Being subject to the piezoelectric force and Coulomb friction, the piezoelectric actuator can move rapidly. Equations of motion are derived based on piezoelectric properties and Newton's law. Static and kinetic friction effects are incorporated. Stress and strain calculations and piezoelectric equations are included in derivation. Circular plate models with polar coordinates are used to account for piezoelectric properties of buzzers. Experimental results are compared with theoretical results. According to experimental results, the present actuator can move at the speed of $37.5 \mathrm{~mm} / \mathrm{sec}$ and generate the force of $15.4 \mathrm{mN}$.
\end{abstract}

\section{Introduction}

With the development of science and technology in various technical fields, such as aerospace, optics, electronics, and medical engineering, high-precision actuators are required [1-6]. Based on the driving principles, types of precision actuators include electrostrictive, magnetostrictive, artificial muscle actuator, shape memory alloy, photostrictive, and mechanochemical actuators. In the electrostrictive category, piezoelectric actuators are small and possess nanoscale displacement resolution and large driving force [7].

The piezoelectric material used in a piezoelectric actuator comes in types of multilayer, unimorph, bimorph, cylindrical, ring, and disc forms [8-14]. By means of impact drive force, moving bodies can be driven by impulse force [15-17] or by alternate stick and slip method [18-24]. Piezoelectric actuators are employed in precision platforms, atomic force microscopes [25], mobile phones, and digital camera lens drives.

To achieve innovative and diverse actuator applications, this study focused on actuators capable of large displacement, for which two piezoelectric buzzers subject to both piezoelectric force and Coulomb friction are employed to undergo displacement. Unlike the literature, piezoelectric buzzers constitute the driving element in proposed actuators. With a proper driving voltage and duty ratio, the piezoelectric buzzers generate force and velocity, enabling the actuator to move. This study derives theoretical models based on piezoelectric material properties, buzzer disk geometry, stress and strain relationships, and Coulomb friction. Actuator characteristics, including the velocity and force, are measured to validate theoretical models.

\section{Actuator Design and Actuation Principle}

As depicted in Figure 1, the proposed actuator consists of a moving body, a rod, a driving source, a fixed rod, and a base. The moving body comprises a load, upper part, lower part, four screws, and four rubbers. The driving source comprises two piezoelectric buzzers and a connective ring. Figure 2 shows an exploded view of the piezoelectric actuator. Table 1 shows material properties of the actuator. Screws between the moving body and the rod are adjusted in order to generate appropriate preloads and Coulomb friction so as to expedite movement.

Piezoelectric buzzers consist of a metal layer and a piezoelectric layer. Both layers are pasted together. Alternating currents result in both extension and shrink states such that both buzzers become either convex or concave geometry, as depicted in Figure 3. The actuation force in this study comes from both buzzers. To find out which driving type has faster 
TABLE 1: Material properties of piezoelectric actuator.

\begin{tabular}{|c|c|c|c|}
\hline & Material & Mass (mg) & Dimensions (mm) \\
\hline Piezoelectric buzzer & Piezoelectric element and metal & 65 & $\begin{array}{c}\text { Piezoelectric element: } D 9 \times t 0.08 \\
\text { Metal: } D 12 \times t 0.05\end{array}$ \\
\hline Connective ring & Plastic & 52 & $D 14 \times d 11 \times t 1$ \\
\hline Rod & Carbon fiber & 67 & $D 1.1 \times L 25$ \\
\hline Fixed rod & Carbon fiber & 13 & $D 2 \times L 6.5$ \\
\hline Moving body & - & 466 & - \\
\hline Upper part & Metal & 80 & - \\
\hline Lower part & Metal & 80 & - \\
\hline Rubber & Rubber & 10 & - \\
\hline Load & Aluminum alloy & 186 & - \\
\hline
\end{tabular}

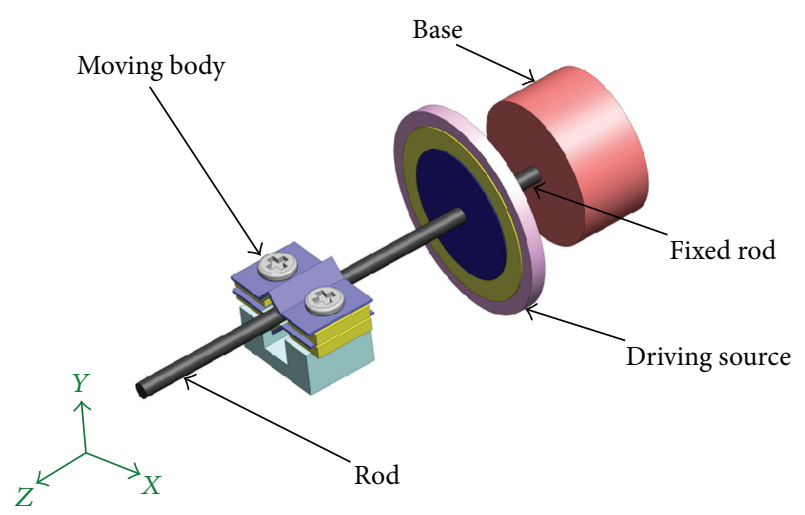

FIgURE 1: Piezoelectric actuator.

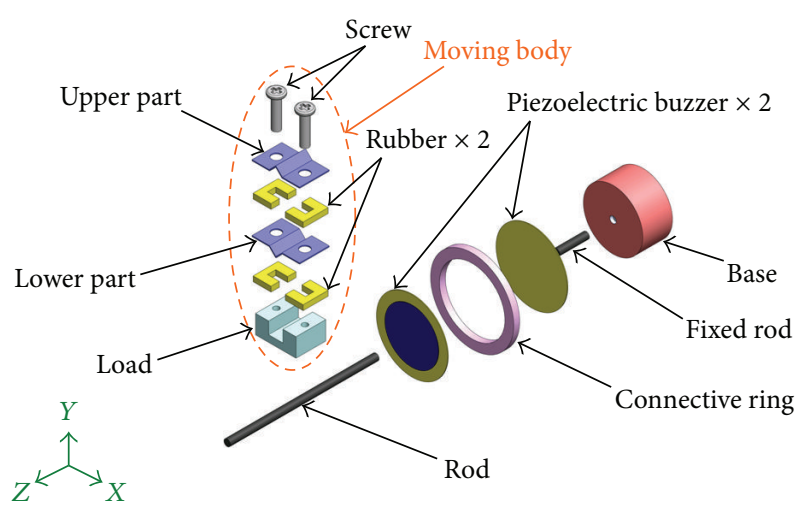

FIGURE 2: Exploded view of piezoelectric actuator.

velocity and larger force, this study designs and compares two kinds of piezoelectric actuators. As depicted in Figure 3(a), both buzzers in actuator A are subject to the same voltage as a parallel circuit. By contrast, both buzzers in actuator $\mathrm{B}$ are subject to opposite sign voltages as a series circuit. The deformation speed of the piezoelectric buzzer varies with the duty ratio. As depicted in Figure 3, being subject to alternating currents, actuator A deforms more severely than actuator B. Figure 4 depicts the principle of actuator translation motion. Figures 4(a) and 4(b) show the driving procedures of actuators $\mathrm{A}$ and $\mathrm{B}$, respectively. The deformed directions of the piezoelectric buzzer and piezoelectric force are in the same direction.

Concerning the driving procedure of actuator A: the moving body is initially stationary (Step 1). When the two piezoelectric buzzers slowly expand simultaneously, the summation of the piezoelectric and inertial forces is smaller than that of the static friction (Step 2); thus, the moving body and rod move together to the left. When both piezoelectric buzzers rapidly shrink, the piezoelectric force becomes greater than the kinetic friction (Step 3). This results in displacement of the moving body. At last, two piezoelectric buzzers slowly return to their undeformed state and the moving body moves to the left with the rod (Step 4). At this moment, the moving body has made a small displacement with respect to the original position in a fixed $X Y Z$ coordinate system. Repeating (Step 1) to (Step 4), the actuator keeps moving to the left direction. On the contrary, if two piezoelectric buzzers rapidly expand first before they slowly shrink, the actuator moves to the right direction.

The driving procedure of actuator $\mathrm{B}$ is as follows. The moving body is initially stationary (Step 1). When the right piezoelectric buzzer slowly shrinks and the left piezoelectric buzzer slowly expands at the same time, the summation of the piezoelectric and inertial forces is smaller than that of the static friction (Step 2); thus, the moving body and rod move together to the left. When the right piezoelectric buzzer rapidly expands while the left piezoelectric buzzer rapidly shrinks simultaneously, the piezoelectric force becomes greater than the kinetic friction. This leads to displacement of the moving body (Step 3). At last, two piezoelectric buzzers slowly return to their undeformed state and the moving body moves to the left with the rod (Step 4). At this moment, the moving body has made a small displacement with respect to the original position in the fixed $X Y Z$ coordinate system. Repeating (Step 1) to (Step 4), actuator B keeps moving to the left direction. On the contrary, if the right buzzer rapidly shrinks while the left buzzer rapidly expands, the actuator moves to the right direction.

\section{Theoretical Derivation}

This paper presents actuator of two types: actuator A and actuator B. Both are different in voltage polarity. It will be later 

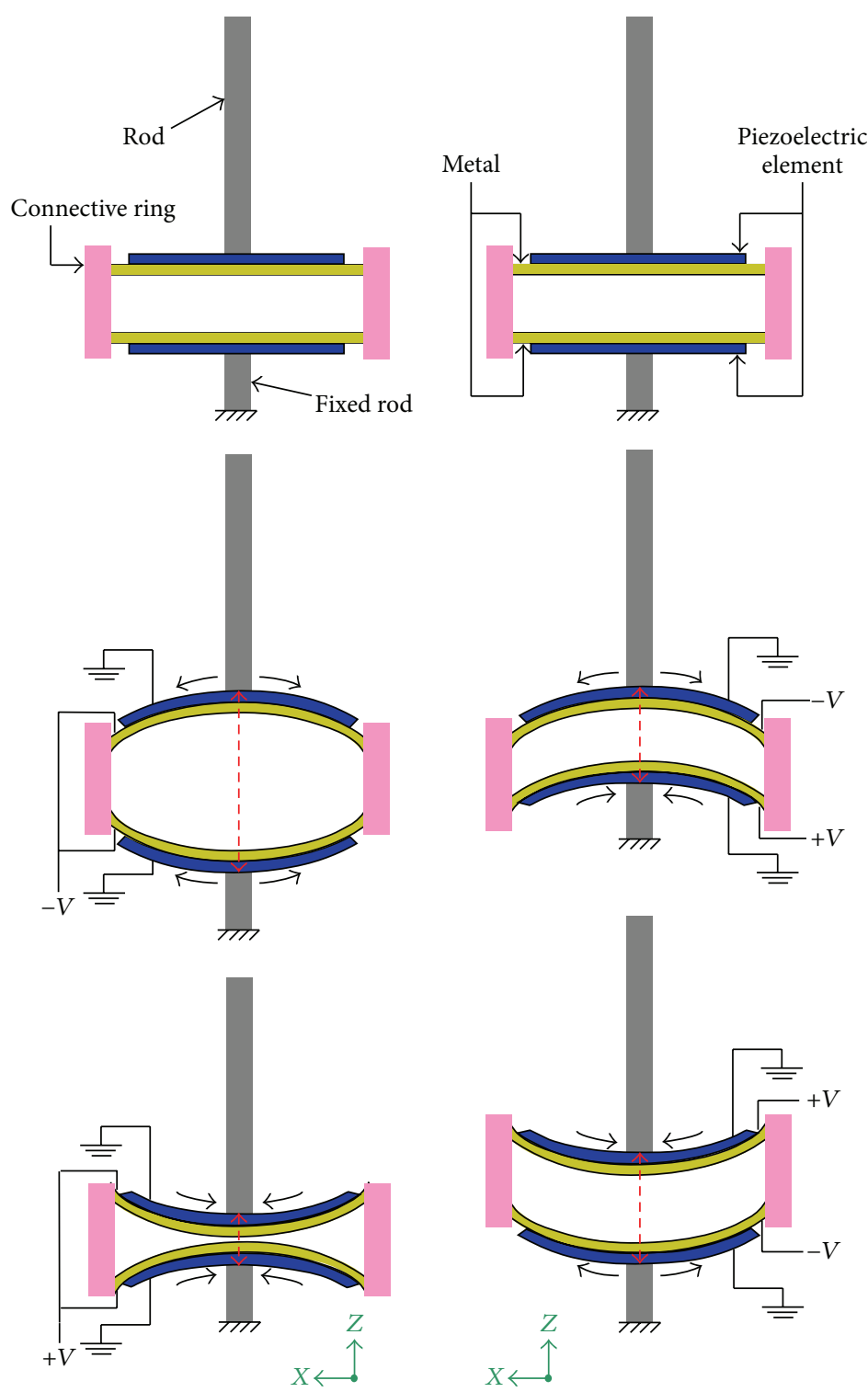

(a)

(b)

FIGURE 3: Driving voltage and deformation direction of (a) actuator A and (b) actuator B. The length change of double arrows in (a) is larger than that in (b), which means being subject to alternating currents buzzers in actuator A which deforms more severely than actuator B.

described in another section on experiments that actuator A moves faster than actuator B. Therefore, this study only derives the dynamic equations of actuator A. Based on the dynamic equation, this study calculates actuator force and velocity.

The mechanical model of proposed piezoelectric actuators is depicted in Figure 5. Assume that the rim of a piezoelectric buzzer is fixed but the center can vibrate freely. When the driving voltage is applied to the buzzer, the buzzer center deforms. As the voltage is applied to the actuator, both buzzers produce forces to the right, making the rod move to the right. When kinetic friction occurs between the rod and the moving body, the moving body moves to the right.
Being subject to two piezoelectric forces $F_{p}$ generated by two buzzers and friction force $F_{f}$, the equation of motion is written as

$$
\left(2 m_{p}+m_{r}+m_{c}+m_{m}\right) \ddot{z}+2 c_{p} \dot{z}+2 k_{p} z=2 F_{p}-F_{f}
$$

where $m_{p}$ is the buzzer mass, $m_{r}$ is the rod mass, $m_{c}$ is the connective ring mass, $m_{m}$ is the mass of the moving body, $c_{p}$ is the damping coefficient of the piezoelectric buzzer, $k_{p}$ is the stiffness coefficient of the buzzer, $F_{p}$ is the piezoelectric force generated by the buzzer, $F_{f}$ is the friction force, $\ddot{z}$ is the moving body acceleration, $\dot{z}$ is the moving body velocity, and $z$ is the moving body displacement. 


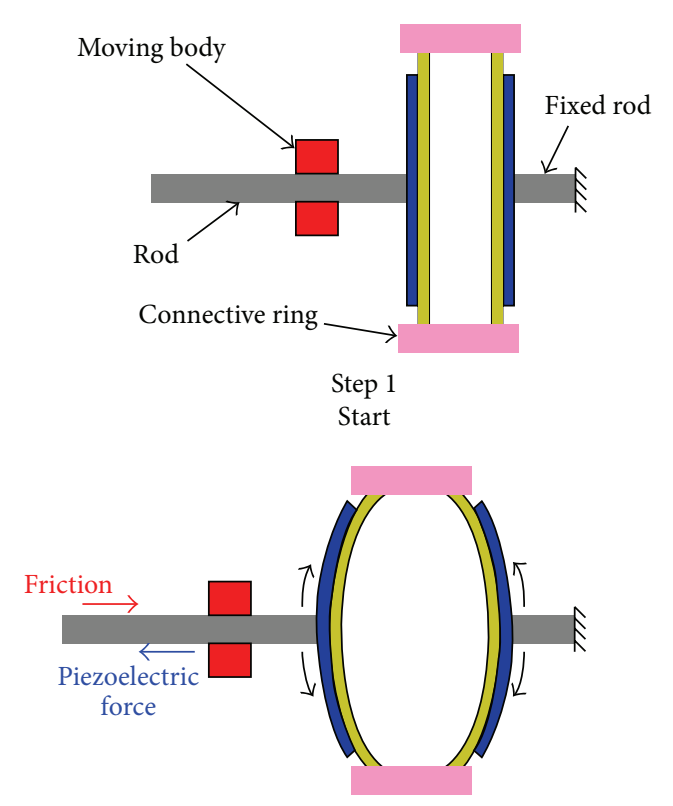

Step 2

Slow deformation

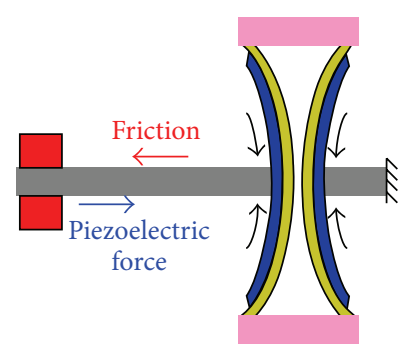

Step 3

Rapid deformation

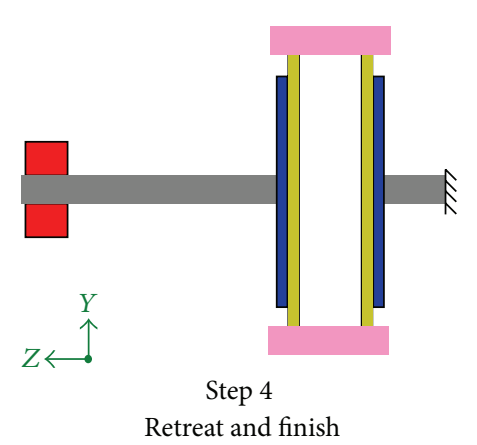

(a)
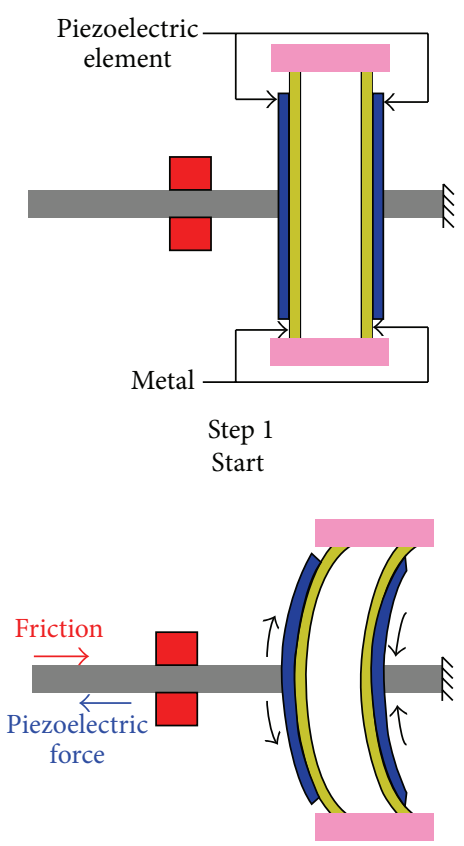

Step 2

Slow deformation

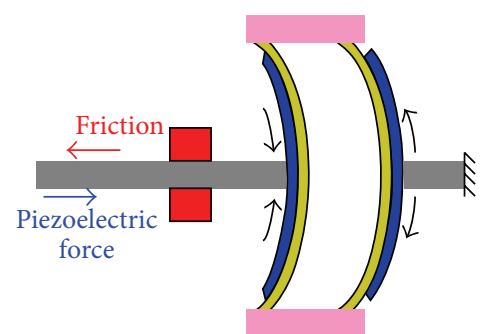

Step 3

Rapid deformation

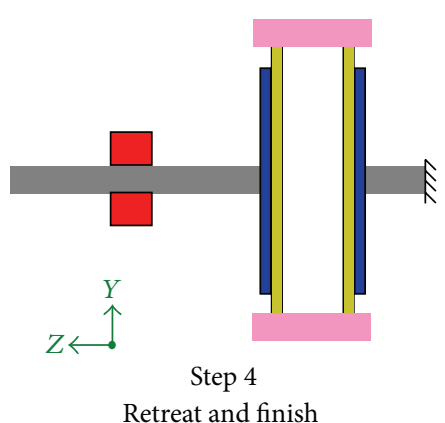

(b)

FIGURE 4: Driving procedures of (a) actuator A and (b) actuator B.

If a concentrated loading $P$ is applied to the buzzer disk center, the shear force $Q_{r}$ at the disk rim and the loading $P$ are equal but in the opposite directions. Hence,

$$
2 \pi r Q_{r}=-P .
$$

According to the theory of plates and shells [26], the shear force is expressed by

$$
Q_{r}=-D \frac{d}{d r}\left(\nabla^{2} \omega\right)=-D \frac{d}{d r}\left(\frac{d^{2} \omega}{d r^{2}}+\frac{1}{r} \frac{d \omega}{d r}+\frac{1}{r^{2}} \frac{d^{2} \omega}{d \theta^{2}}\right),
$$



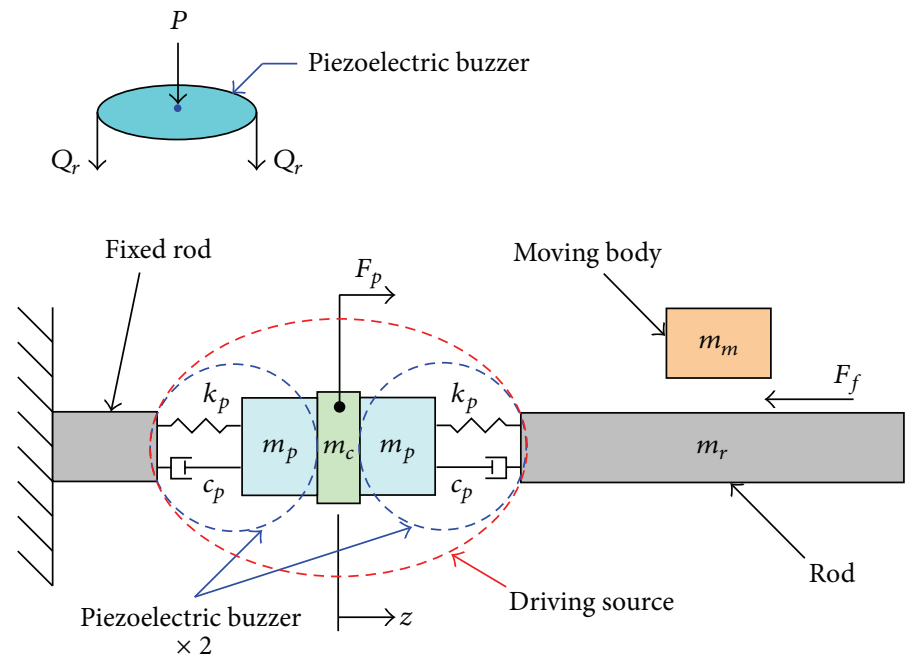

FIGURE 5: Mechanical model of the present piezoelectric actuator.

where $\omega$ denotes the buzzer deformation in the axial direction and $D$ denotes the bending stiffness. Substituting (3) into (2) gives

$$
\frac{d}{d r}\left(\nabla^{2} \omega\right)=\frac{d}{d r}\left(\frac{1}{r} \frac{d}{d r} r \frac{d \omega}{d r}\right)=-\frac{Q_{r}}{D}=\frac{P}{2 \pi r D} .
$$

Integrating this equation three times gives

$$
\omega=\frac{P}{8 \pi D}\left(r^{2} \log r+C_{1} r^{2}+C_{2} \log r+C_{3}\right),
$$

where $C_{1}, C_{2}$, and $C_{3}$ are constants of integration. When the buzzer rim with radius $a$ is fixed, the first boundary condition is written as

$$
\frac{d \omega}{d r}=0, \quad \text { when } r=0
$$

and two other boundary conditions are written as

$$
\omega=\frac{d \omega}{d r}=0, \quad \text { when } r=a .
$$

Substituting (5) into (6) yields unknown constants

$$
C_{1}=-\frac{1}{2}-\log a, \quad C_{2}=0, \quad C_{3}=\frac{1}{2} a^{2} .
$$

Substituting (8) into (5) yields the buzzer deformation

$$
\omega=\frac{P}{8 \pi D}\left[\frac{1}{2}\left(a^{2}-r^{2}\right)+r^{2} \log \frac{r}{a}\right] .
$$

The radial moment $M_{r}$ and the tangential moment $M_{\theta}$ are, respectively, written as [26]

$$
\begin{aligned}
& M_{r}=-D\left(\frac{d^{2} \omega}{d r^{2}}+\frac{\nu}{r} \frac{d \omega}{d r}\right), \\
& M_{\theta}=-D\left(\frac{1}{r} \frac{d \omega}{d r}+\nu \frac{d^{2} \omega}{d r^{2}}\right),
\end{aligned}
$$

where $v$ is the Poisson's ratio. Substituting (9) into (10) gives the moments

$$
\begin{aligned}
& M_{r}=\frac{P}{4 \pi}\left[(1+\nu) \log \frac{a}{r}-1\right], \\
& M_{\theta}=\frac{P}{4 \pi}\left[(1+\nu) \log \frac{a}{r}-\nu\right] .
\end{aligned}
$$

The radial stress $\sigma_{r}$ and the tangential stress $\sigma_{\theta}$ are, respectively, written as [26]

$$
\begin{gathered}
\sigma_{r}=-\frac{6 M_{r}}{t^{2}}, \\
\sigma_{\theta}=-\frac{6 M_{\theta}}{t^{2}},
\end{gathered}
$$

where $t$ is the thickness. Substituting (11) into (12) yields stresses

$$
\begin{aligned}
& \sigma_{r}=\frac{-6 P}{4 \pi t^{2}}\left[(1+v) \log \frac{a}{r}-1\right], \\
& \sigma_{\theta}=\frac{-6 P}{4 \pi t^{2}}\left[(1+v) \log \frac{a}{r}-v\right] .
\end{aligned}
$$

According to Hooke's law, the radial strain $\varepsilon_{r}$ and the tangential strain $\varepsilon_{\theta}$ are, respectively, written as

$$
\begin{gathered}
\varepsilon_{r}=\frac{1}{E}\left(\sigma_{r}-\nu \sigma_{\theta}\right), \\
\varepsilon_{\theta}=\frac{1}{E}\left(\sigma_{\theta}-\nu \sigma_{r}\right) .
\end{gathered}
$$

Substituting (13) into (14) gives

$$
\begin{gathered}
\varepsilon_{r}=\frac{-6 P}{4 \pi E t^{2}}\left[(1+\nu)(1-v) \log \frac{a}{r}-\left(1-\nu^{2}\right)\right], \\
\varepsilon_{\theta}=\frac{-6 P}{4 \pi E t^{2}}\left[(1+\nu)(1-\nu) \log \frac{a}{r}\right] .
\end{gathered}
$$




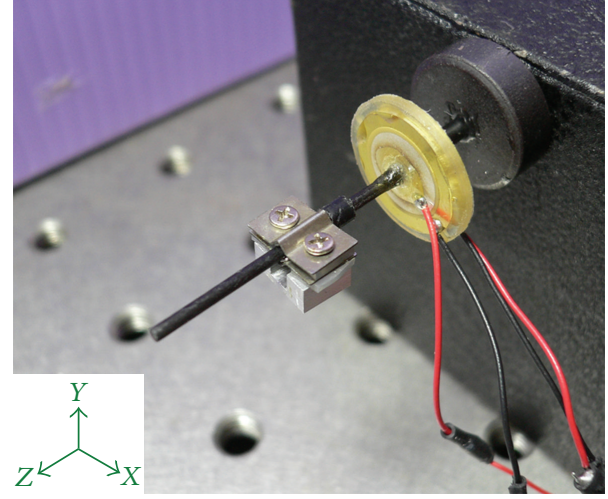

Figure 6: Photograph of piezoelectric actuator.

Dealing with circular plates, the piezoelectric equation can be written as [27]

$$
\varepsilon_{r}=s_{11}^{E} \sigma_{r}+s_{12}^{E} \sigma_{\theta}+s_{13}^{E} \sigma_{z}+d_{31} E_{z}
$$

where $s_{11}^{E}, s_{12}^{E}$, and $s_{13}^{E}$ denote elasticity constants of the buzzer, $d_{31}$ denotes the charge constant of the buzzer, $\sigma_{z}$ denotes the axial stress, and $E_{z}$ denotes the exerted electric field. The piezoelectric force and the electric field are, respectively, written as [28]

$$
\begin{gathered}
F_{p}=\sigma_{z} A_{1}, \\
E_{z}=\frac{V}{t},
\end{gathered}
$$

where $A_{1}$ denotes the buzzer disk area and $V$ the exerted electric voltage. Substituting (13), (15), and (17) into (16) yields the piezoelectric force

$$
F_{p}=\frac{A_{1}}{S_{13}^{E}}\left(\varepsilon_{r}-s_{11}^{E} \sigma_{r}-s_{12}^{E} \sigma_{\theta}-d_{31} \frac{V}{t}\right) .
$$

The friction force between the moving body and rod can be expressed by [29]

$$
F_{f}(\dot{z})=\gamma_{1}\left(\tanh \left(\gamma_{2} \dot{z}\right)-\tanh \left(\gamma_{3} \dot{z}\right)\right)+\gamma_{4} \tanh \left(\gamma_{5} \dot{z}\right)+\gamma_{6} \dot{z} .
$$

Based on (19), the static friction coefficient can be approximated by the term $\gamma_{1}+\gamma_{4}$. The term $\tanh \left(\gamma_{2} \dot{z}\right)-\tanh \left(\gamma_{3} \dot{z}\right)$ captures the Stribeck effect where the friction coefficient decreases from the static friction coefficient with the increasing slip velocity. The third term $\gamma_{4} \tanh \left(\gamma_{5} \dot{z}\right)$ accounts for the Coulomb friction effect. The last term $\gamma_{6} \dot{z}$ accounts for the viscous damping effect. Finally, substituting (18) and (19) into (1) yields the dynamic equation. MATLAB/Simulink softwares are in turn executed to verify these equations. Comparisons of theory and experimental results are performed.

\section{Experimental Results}

Figure 6 shows the photograph of the piezoelectric actuator in this study. In system identification, the driving voltage

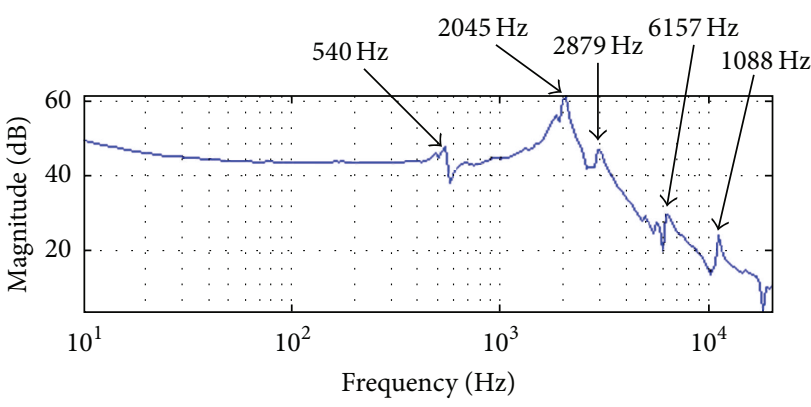

(a)

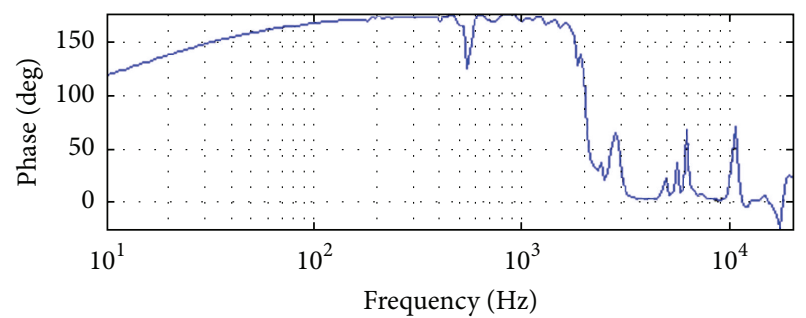

(b)

Figure 7: Bode plot.

is a $2 \mathrm{~V}$ sinusoidal wave within the scanning range of 10 to $20 \mathrm{kHz}$. The driving voltage of the actuator driver is adjusted to drive the piezoelectric actuator. The vibrometer emits laser beams to detect the vibration displacement of the piezoelectric buzzer, passing the signal value of the vibration displacement to the vibrometer controller. Finally, vibration data stored in the vibrometer controller are converted into a Bode plot. Figure 7 depicts the Bode plot, where resonant frequencies include $540 \mathrm{~Hz}, 2045 \mathrm{~Hz}, 2879 \mathrm{~Hz}, 6157 \mathrm{~Hz}$, and $10887 \mathrm{~Hz}$. After velocity measurements for all the natural frequency, experimental results exhibited that actuator at frequency $2045 \mathrm{~Hz}$ obtained the fastest velocity and at frequency $6157 \mathrm{~Hz}$, obtained the second fastest velocity. The purpose of the modal analysis in Figure 8 is to obtain the modal shapes rather than natural frequencies. Natural frequencies of Bode plot are actually driving frequencies of actuator in velocity and force experiments. According to the finite element modal analysis result, the first ten natural frequencies of the actuator are $324.2 \mathrm{~Hz}, 324.9 \mathrm{~Hz}, 1650 \mathrm{~Hz}$, $2585 \mathrm{~Hz}, 2592 \mathrm{~Hz}, 4508 \mathrm{~Hz}, 5773.6 \mathrm{~Hz}, 5774.4 \mathrm{~Hz}, 7806.7 \mathrm{~Hz}$, and $10969 \mathrm{~Hz}$. Figure 8 depicts modal shapes at $1650 \mathrm{~Hz}$ and $4508 \mathrm{~Hz}$. Figure 8(a) shows that two buzzers simultaneously deform in opposite directions, corresponding to the mode shape of actuator A. Figure 8(b) shows that both buzzers simultaneously deform in the same direction, corresponding to the mode shape of actuator B.

Velocity measurements and force measurements experiments are carried out for comparison between actuators $\mathrm{A}$ and B. According to resonant peaks, driving frequencies selected for both actuators are $2045 \mathrm{HZ}$ and $6157 \mathrm{~Hz}$, respectively. When the driving voltage employs rectangular waves [21] to drive the piezoelectric buzzer, according to the excitation frequency and duty ratio of the driving voltage, the piezoelectric buzzer deforms and produces an output 

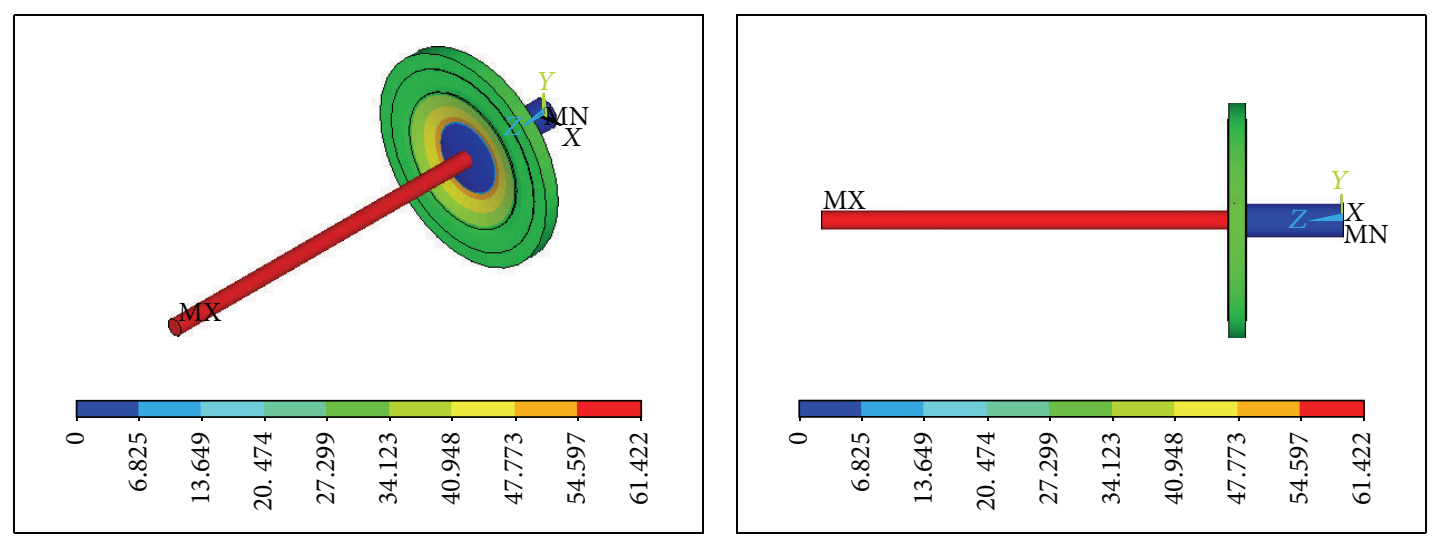

(a)
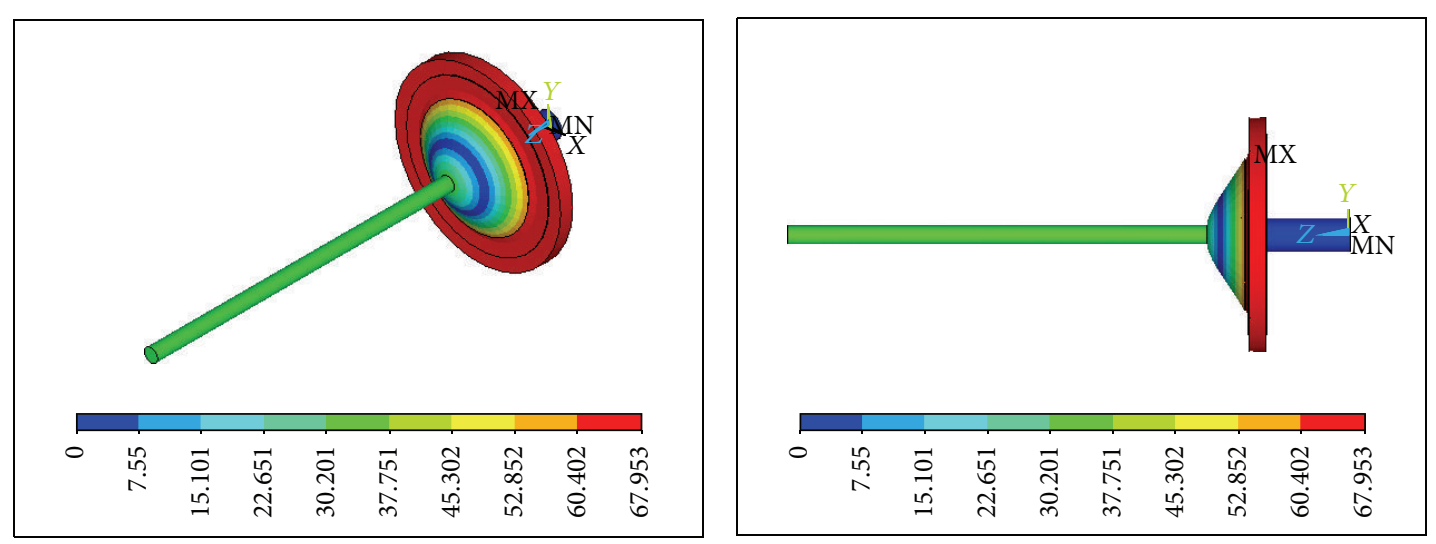

(b)

FIGURE 8: Actuator mode shapes obtained from the finite element analysis: (a) $1650 \mathrm{~Hz}$ whose deformation in mode shape occurs in the rod and (b) $4508 \mathrm{~Hz}$ whose deformation in mode shape occurs on the rim.

wave resembling a sawtooth wave. The duty ratio of the driving voltage rectangular waveforms is adjusted in this study to control the sawtooth proportion and the sawtooth wave direction [22]. Changes in the duty ratio influence the movement direction and velocity. Figure 9 shows a schematic diagram of the driving voltage, buzzer deformation, and actuator displacement. As depicted in Figure 9(a), $W / T$ ratio is called the duty ratio, defined as the time percentage of high potential signal in a period, where $W$ is the time interval of high potential signal and $T$ is period. According to the experimental verification, $W / T$ ratio of the driving voltage affects the velocity of the moving body. As depicted in Figure 9(b), in each period of the sawtooth signal, the slope of the first half is smaller than that of the second half. Therefore, in the first half, the static friction exists between the moving body and the rod, and no relative displacement occurs between both of them. In the second half, however, the slope is larger, and the dynamic friction between the moving body and rod allows the moving body to move for a short distance. As a consequence, persistent switching between static and dynamic friction causes the moving body to move, as shown in Figure 9(c). For the actuator velocity measurement, Figure 10 shows the experimental setup, which includes a vibrometer controller (Polytec OFV 3001), vibrometer (Polytec OFV 512), waveform generator
(Agilent 33210A), and actuator driver (Echo ENP-4012B). In experiments, firstly, the waveform generator is used to generate the rectangular voltage waveform with a duty ratio. The driving voltage of the actuator driver is adjusted so as to drive the actuator. The vibrometer is used to measure the actuator displacement and velocity, which are in turn transmitted to the vibrometer controller.

Figure 11 compares experimental and simulation results, in which the driving voltage is $40 \mathrm{~V}$ for both actuators and driving frequencies are $2045 \mathrm{HZ}$ and $6157 \mathrm{~Hz}$ for actuators A and $\mathrm{B}$, respectively. When the duty ratio is prescribed as $50 \%$, the buzzer deforms in an isosceles triangle waveform and, the moving velocity of the moving body is difficult to control and stabilize; thus, a duty ratio of $50 \%$ is not appropriate. According to experimental results, $10 \%$ and $90 \%$ duty ratios have little effect on velocity; thus, results of both duty ratios are not included in comparison. Figure 11 also shows that when the duty ratio is from $20 \%$ to $40 \%$ and from $60 \%$ to $80 \%$, the moving bodies moved in opposite directions. The fastest speed is obtained at duty ratios of $40 \%$ and $60 \%$. Actuator A moves faster than actuator B. Experimental results are consistent with simulation at $40 \%$ and $60 \%$ duty ratios, as depicted in Figure 11.

Figure 12 shows that the moving velocity is proportional to the driving voltage. Actuator A moves obviously faster 


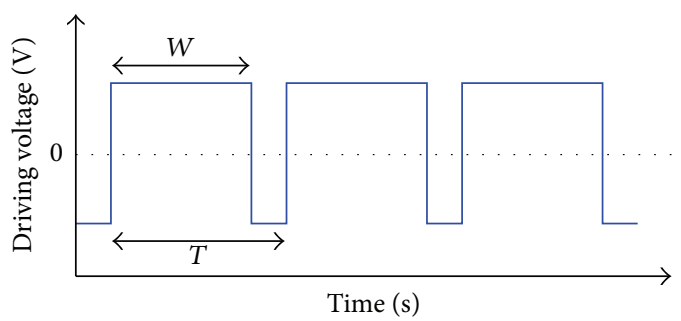

(a)

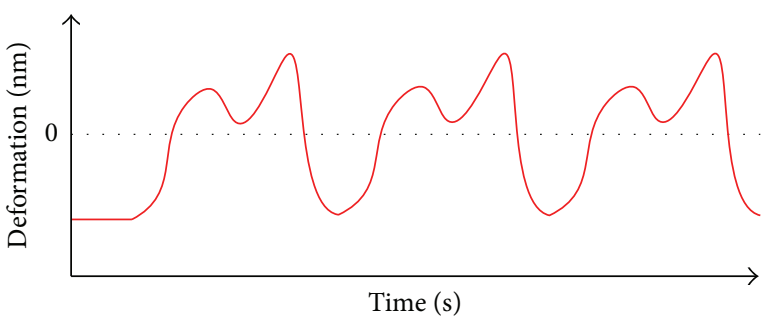

(b)

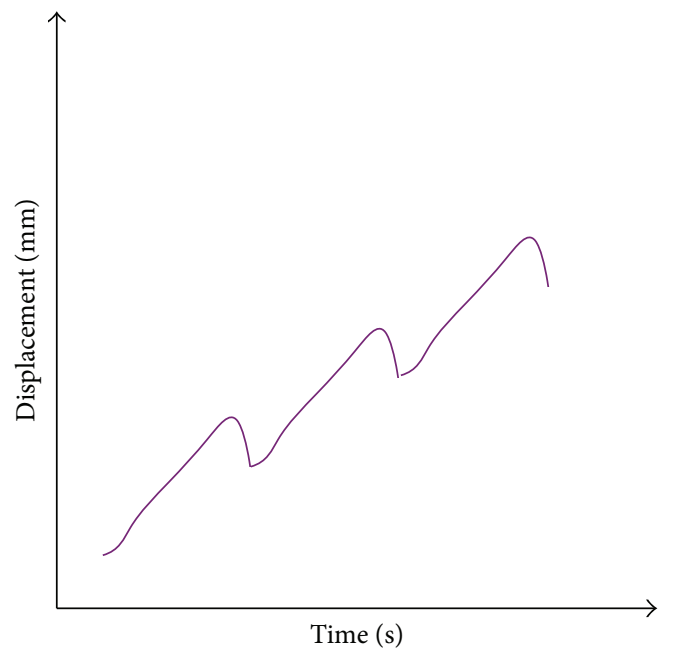

(c)

FIGURE 9: Schematic diagrams of (a) driving voltage with duty ratio $=W / T$, (b) deformation of piezoelectric buzzer in actuator, and (c) actuator displacement.

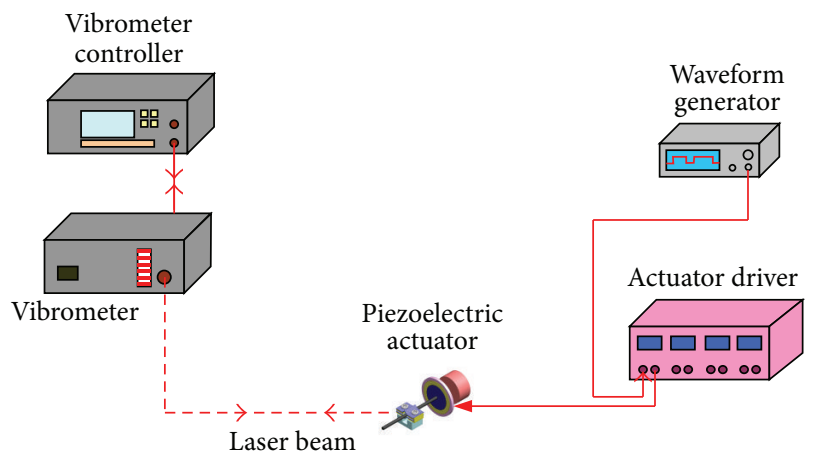

FIGURE 10: Experimental setup for the actuator velocity measurement.

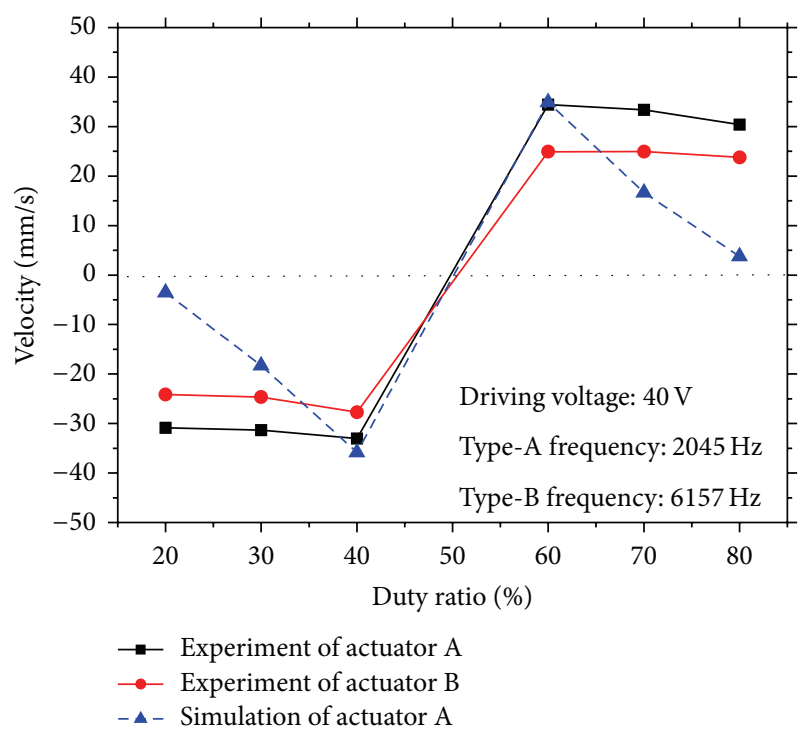

FIGURE 11: Comparison of experimental and simulation results in velocity variation with duty ratios.

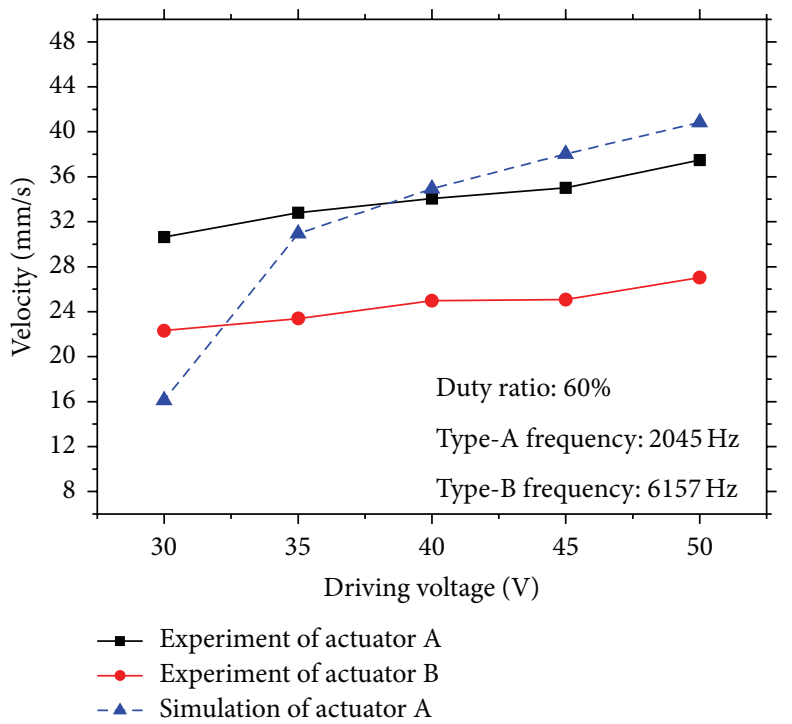

FIGURE 12: Comparison of experimental and simulation results in translational velocity.

than actuator B. Moreover, actuators A and B subject to $50 \mathrm{~V}$ driving voltage in experiments move at $37.5 \mathrm{~mm} / \mathrm{sec}$ and $27.02 \mathrm{~mm} / \mathrm{sec}$, respectively. In contrast, actuator A in simulation subject to $50 \mathrm{~V}$ driving voltage moves at $40.82 \mathrm{~mm} / \mathrm{sec}$. Although the maximum distance traveled is $20 \mathrm{~mm}$, if no space constraint, the distance traveled by the moving body is not limited.

Figure 13 shows that the force is proportional to the driving voltage. The force experiments of actuator $\mathrm{A}$ and actuator B under a driving voltage of $50 \mathrm{~V}$ can reach $15.4 \mathrm{mN}$ and $11.53 \mathrm{mN}$, respectively. The force simulation of actuator A under a driving voltage of $50 \mathrm{~V}$ is $26.18 \mathrm{mN}$. The measured force from actuator $\mathrm{A}$ is larger than that in actuator B. Measured forces are smaller than calculated forces in simulation 


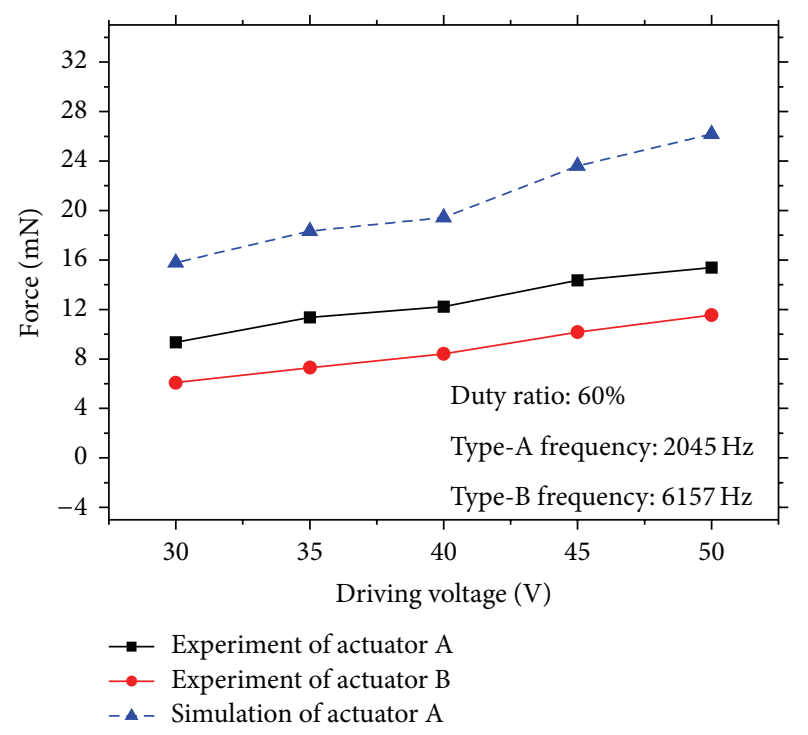

Figure 13: Comparison of experimental and simulation results in force versus voltage.

results due to the imperfect straightness of the rod. According to the overall comparison, actuator A performs superior to actuator B because actuator $\mathrm{A}$ has an advantage in its mode shape that yields larger force and displacement.

\section{Conclusion}

This study has designed, modeled, and measured innovative piezoelectric actuators of dual buzzers, alternately subject to piezoelectric force and Coulomb friction. Although the piezoelectric actuator moves $20 \mathrm{~mm}$ distance in this study, there is no distance limit as long as the rod is lengthened. This is unlike most belief that piezoelectric actuators move precisely but short. Based on experimental results, the present actuator can generate the translational velocity of $37.5 \mathrm{~mm} / \mathrm{sec}$ and the force of $15.4 \mathrm{mN}$. According to experimental results, concerning velocities and forces, actuator A performs better than actuator $\mathrm{B}$ because the design of actuator A leads to larger forces and faster moving velocity. The proposed dynamic model that incorporates both mechanical and piezoelectric properties has been validated by experimental results. Future applications of this type of piezoelectric actuator include the focusing or zooming of the mobile phone cameras, digital cameras, and digital video cameras.

\section{References}

[1] B. Watson, J. Friend, and L. Yeo, "Piezoelectric ultrasonic micro/ milli-scale actuators," Sensors and Actuators A, vol. 152, no. 2, pp. 219-233, 2009.

[2] C. K. Wang, H. P. Huang, and K. Chien, "Piezo-electric driver of ultrasonic motor on the humanoid robot," in Proceedings of the IEEE International Conference on Advanced Robotics and its Social Impacts (ARSO '08), pp. 23-25, August 2008.

[3] K. Uchino, "Piezoelectric actuators 2006," Journal of Electroceramics, vol. 20, no. 3-4, pp. 301-311, 2007.
[4] R. Bansevicius and V. Blechertas, "Multi-degree-of-freedom ultrasonic motors for mass-consumer devices," Journal of Electroceramics, vol. 20, no. 3-4, pp. 221-224, 2008.

[5] T. Morita, "Miniature piezoelectric motors," Sensors and Actuators A, vol. 103, no. 3, pp. 291-300, 2003.

[6] Z. M. Zhang, Q. An, J. W. Li, and W. J. Zhang, "Piezoelectric friction-inertia actuator-a critical review and future perspective," The International Journal of Advanced Manufacturing Technology, vol. 62, no. 5-8, pp. 1-17, 2012.

[7] K. Uchino and J. R. Giniewicz, Micromechatronics, Marcel Dekker, New York, NY, USA, 2003.

[8] P. Liu, Z. Wen, and L. Sun, "An in-pipe micro robot actuated by piezoelectric bimorphs," Chinese Science Bulletin, vol. 54, no. 12, pp. 2134-2142, 2009.

[9] K. J. Lim, S. H. Park, Y. J. Yun et al., "Characteristics of disktype linear ultrasonic motor for application to $\mathrm{x}$-y stage," Journal of Electrical Engineer and Technology, vol. 1, no. 1, pp. 101-105, 2006.

[10] P. Juang and C. Tsai, "Characterization of one-wheeled actuator driven by one piezoelectric element," Measurement, vol. 42, no. 1, pp. 112-118, 2009.

[11] S. Jun, S. Lee, S. Lee, H. Kim, and K. Lee, "Piezoelectric linear motor with unimorph structure by co-extrusion process," Sensors and Actuators A, vol. 147, no. 1, pp. 300-303, 2008.

[12] G. Cheng, P. Li, P. Zeng, J. Dong, and F. Sun, "Piezoelectric pump used in bionic underwater propulsion unit," Journal of Bionic Engineering, vol. 4, no. 3, pp. 159-164, 2007.

[13] X. Zhao, C. Zhang, H. Liu, G. Zhang, and K. Li, "Analysis of hysteresis-free creep of the stack piezoelectric actuator," Mathematical Problems in Engineering, vol. 2013, Article ID 187262, 10 pages, 2013.

[14] D. Sun, S. Wang, J. Sakurai, K. Choi, A. Shimokohbe, and S. Hata, "A piezoelectric linear ultrasonic motor with the structure of a circular cylindrical stator and slider," Smart Materials and Structures, vol. 19, no. 4, Article ID 045008, 9 pages, 2010.

[15] D. Mazeika and P. Vasiljev, "Linear inertial piezoelectric motor with bimorph disc," Mechanical Systems and Signal Processing, vol. 36, no. 1, pp. 110-117, 2013.

[16] S. Kawakita, T. Isogai, N. Ohya, and N. Kawahara, "Multilayered piezoelectric bimorph actuator," in Proceedings of the IEEE International Symposium on Micromechatronics and Human Science, pp. 73-78, October 1997.

[17] H. Zhang, G. Cheng, H. Zhao, P. Zeng, and Z. Yang, "A 2dimensional impact driven precise actuator using piezoelectric bimorphs," Frontiers of Electrical and Electronic Engineering in China, vol. 1, no. 4, pp. 405-409, 2006.

[18] H. Ko, K. Lee, K. Yoo, C. Kang, S. Kim, and S. Yoon, "Analysis of tiny piezoelectric ultrasonic linear motor," Japanese Journal of Applied Physics, vol. 45, no. 5B, pp. 4782-4786, 2006.

[19] H. Ko, S. Kim, S. N. Borodinas, P. E. Vasiljev, C. Kang, and $\mathrm{S}$. Yoon, "A novel tiny ultrasonic linear motor using the radial mode of a bimorph," Sensors and Actuators A, vol. 125, no. 2, pp. 477-481, 2006.

[20] K. Lim, J. Lee, S. Park, S. Kang, and H. Kim, "Fabrication and characteristics of impact type ultrasonic motor," Journal of the European Ceramic Society, vol. 27, no. 13-15, pp. 4159-4162, 2007.

[21] C. Kang, K. Yoo, H. Ko, H. Kim, T. Ko, and S. Yoon, "Analysis of driving mechanism for tiny piezoelectric linear motor," Journal of Electroceramics, vol. 17, no. 2-4, pp. 609-612, 2006.

[22] R. Yoshida, Y. Okamoto, and H. Okada, "Development of smooth impact drive mechanism," The Japan Society for Precision Engineering, vol. 68, no. 4, pp. 536-541, 2002. 
[23] T. Morita, H. Murakami, T. Yokose, and H. Hosaka, "A miniaturized resonant-type smooth impact drive mechanism actuator," Sensors and Actuators A, vol. 178, pp. 188-192, 2012.

[24] T. Nishimura, H. Hosaka, and T. Morita, "Resonant-type smooth impact drive mechanism (SIDM) actuator using a boltclamped Langevin transducer," Ultrasonics, vol. 52, no. 1, pp. 7580, 2012.

[25] J. Hunt and B. Bhushan, "Device level studies of adaptive optics sliding components in microprojectors," Microsystem Technologies, vol. 18, no. 1, pp. 137-148, 2012.

[26] S. Timoshenko and S. Woinowsky-Krieger, Theory of Plates and Shells, McGraw-Hill, New York, NY, USA, 1959.

[27] X. Z. Zhang, M. Veidt, and S. Kitipornchai, "Transient bending of a piezoelectric circular plate," International Journal of Mechanical Sciences, vol. 46, no. 12, pp. 1845-1859, 2004.

[28] M. Deshpande and L. Saggere, "An analytical model and working equations for static deflections of a circular multi-layered diaphragm-type piezoelectric actuator," Sensors and Actuators A, vol. 136, no. 2, pp. 673-689, 2007.

[29] C. Makkar, W. E. Dixon, W. G. Sawyer, and G. Hu, "A new continuously differentiable friction model for control systems design," in Proceedings of the IEEE/ASME International Conference on Advanced Intelligent Mechatronics (AIM '05), pp. 600605, July 2005. 


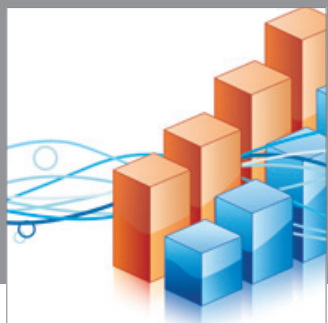

Advances in

Operations Research

mansans

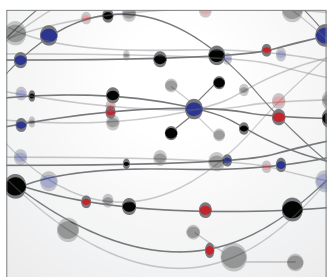

The Scientific World Journal
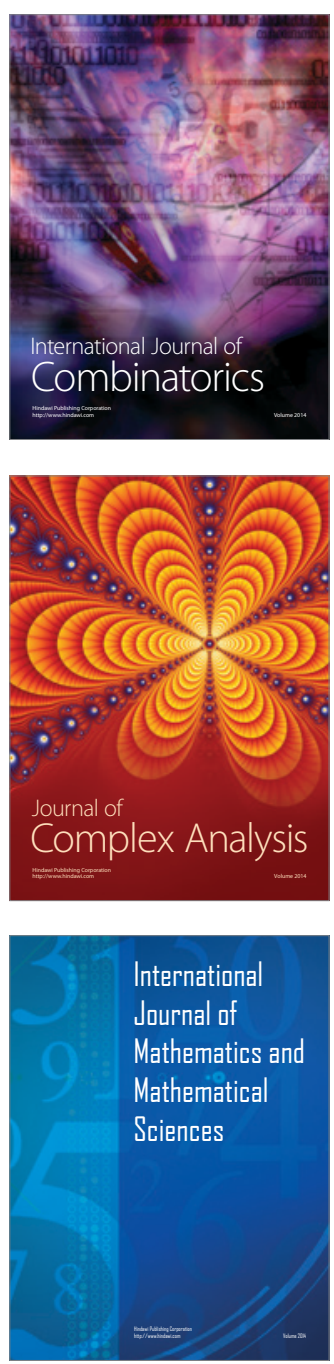
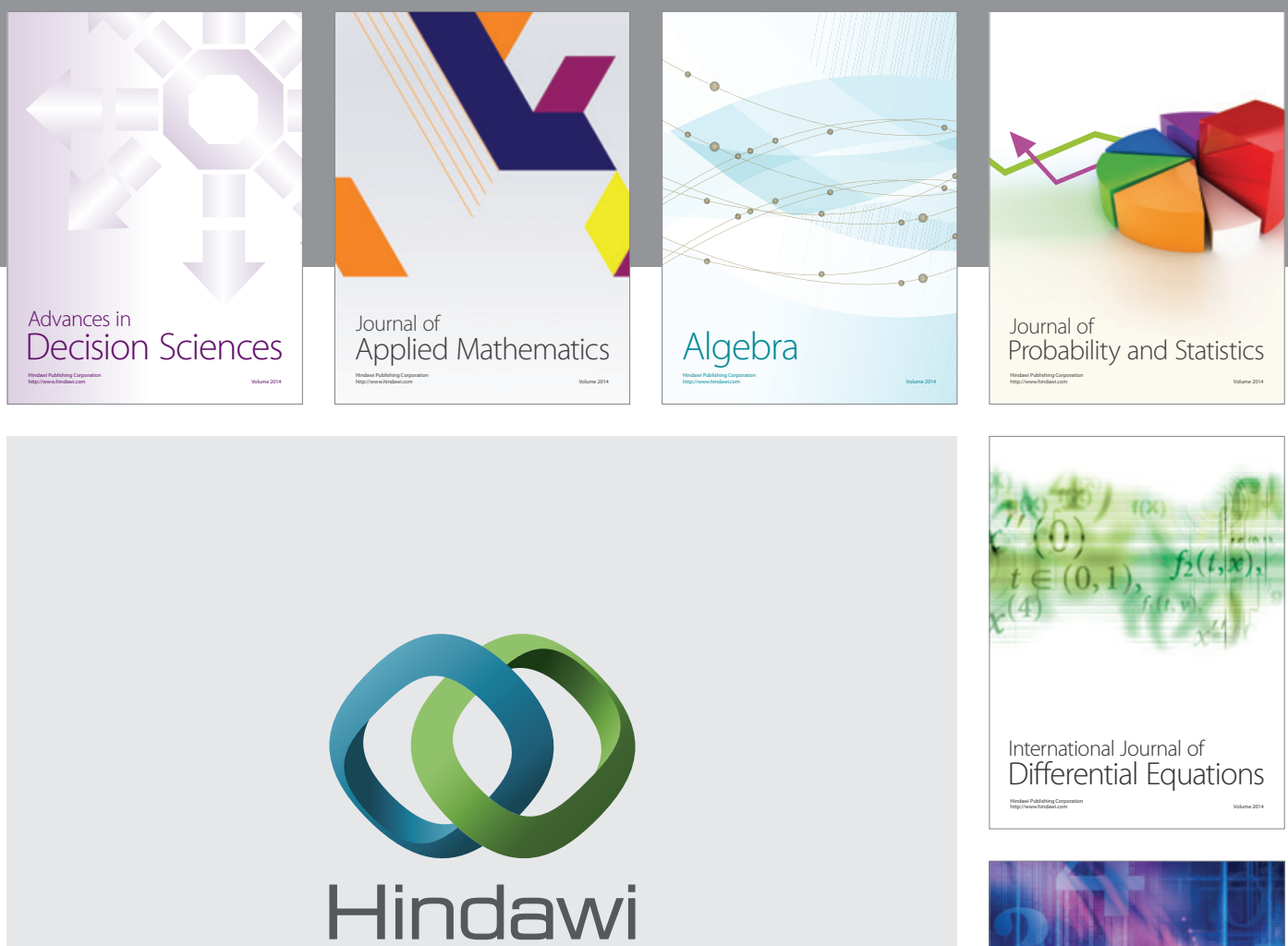

Submit your manuscripts at http://www.hindawi.com
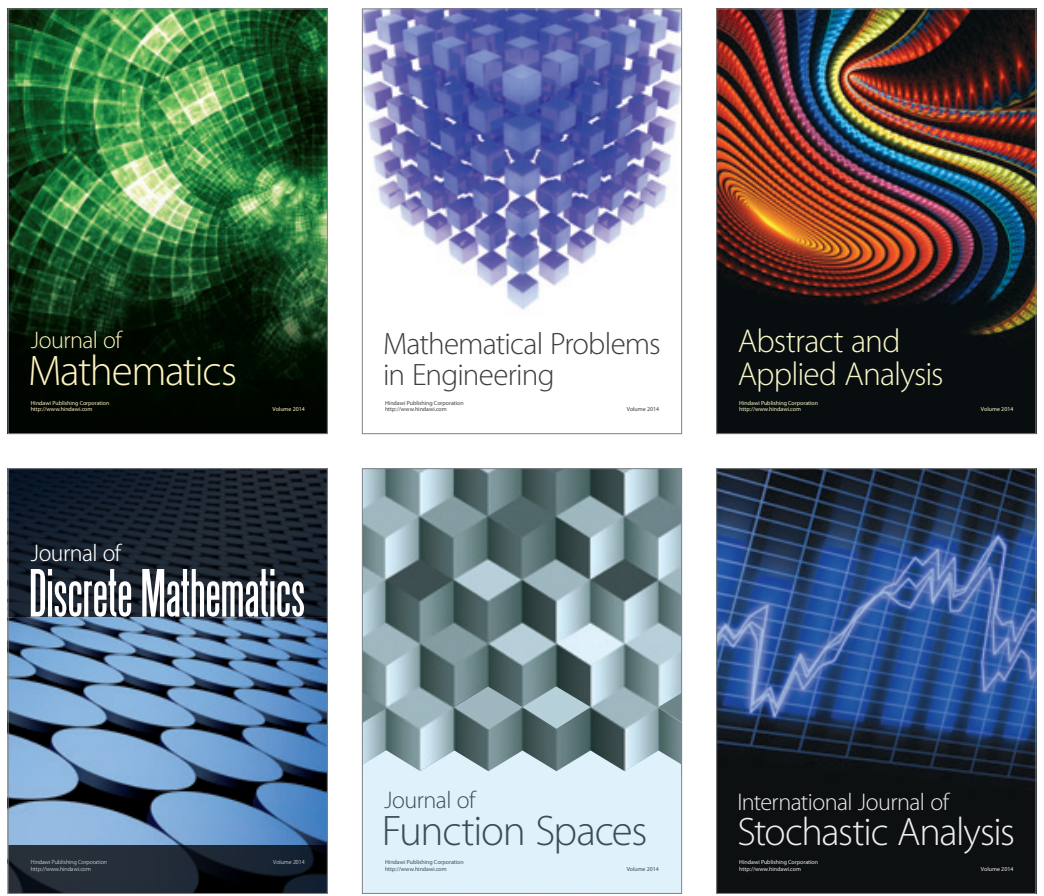

Journal of

Function Spaces

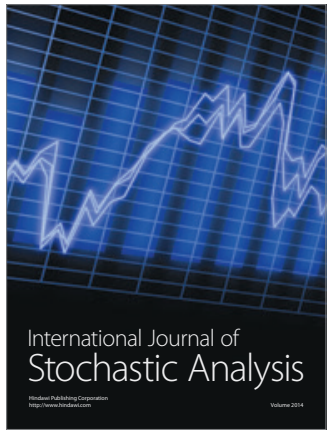

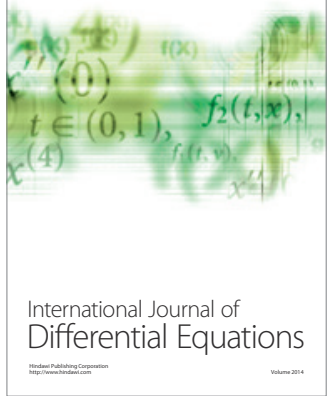
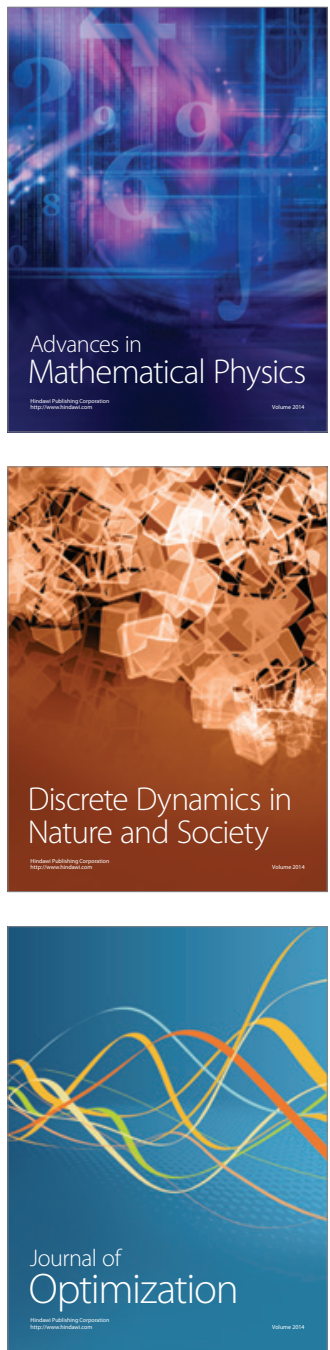